\title{
Réception des cours magistraux avec support projeté en contexte universitaire
}

\author{
Dufour, S. \\ Laboratoire ICAR - Université Lumière Lyon 2 \\ Sophie.Dufour@univ-lyon2.fr
}

\section{Introduction}

Les cours magistraux sont mixtes, utilisant à la fois l'oral et l'écrit. Comme dans d'autres formes d'enseignement universitaire, de nouveaux écrits y prennent une place de plus en plus importante. Il s'agit des genres d'écrits spécifiques générés en particulier par les $\mathrm{TICE}^{1}$ et les logiciels de type PowerPoint (désormais PPT). Ces supports ont une influence sur l'activité des étudiants exposés à ce double flux d'informations écrites et orales. Cette contribution cherche d'une part à mieux observer le fonctionnement dynamique de ces combinaisons synchrones oralographiques, et, d'autre part, à en percevoir les conséquences sur le travail de réception et de stabilisation, par les étudiants, de l'information ainsi doublement délivrée.

On mesure mal actuellement l'impact de ces formes complexes d'enseignement sur la réception des cours et les pratiques d'apprentissage par les étudiants. On peut faire l'hypothèse que dans la majorité des cas la technologie est utilisée comme une commodité sans véritable réflexion méthodologique sur ses usages ${ }^{2}$. Or il est important de s'interroger sur l'intérêt didactique de ces innovations, et sur la méthodologie d'enseignement-apprentissage qui pourrait/devrait les accompagner.

A cet effet, une enquête a été menée en avril 2008 avec pour objectif celui de commencer à explorer l'impact de "nouvelles" formes d'enseignement (introduction des environnements numériques) sur la réception des cours par les étudiants dans ces situations de « dédoublement » discursif. C'est un étudiant de Master 2 de didactique du fle qui a conduit des entretiens enregistrés semi-directifs auprès de 26 étudiants issus de cursus variés (musicologie, lettres modernes (4), psychologie (3), anglais, gestion administrative et commerciale, droit (2), biologie, anthropologie (4), histoire et sciences politiques, espagnol, histoire de l'art, économie-gestion, cinéma, sciences du langage, infocom-anglais, lea anglaisespagnol, géographie). Le questionnaire élaboré placé en annexe s'emploie à balayer les formes de cours auxquelles les étudiants sont habituellement exposés (TD sans PPT, TD avec PPT, TD avec Rétroprojecteur (RP), cours magistral $(\mathrm{CM})$ vocal, $\mathrm{CM}$ vocal + tableau, $\mathrm{CM}$ vocal + RP, etc.) afin de recueillir les représentations sur les pratiques spontanées qu'ils génèrent. Il s'agit plus précisément dans un premier temps d'apprécier les réactions des interviewés s'agissant des présentations PPT (impression générale ? intérêt ? efficacité ? aide à la prise de notes ?), puis ensuite de l'emploi des TICE dans la mise en ligne qu'elles en proposent pour les étudiants. Le dernier volet de l'entretien permet une meilleure approche des stratégies que les étudiants déclarent avoir pendant les cours en matière de prise de notes et de gestion des différents supports écrits qu'on leur propose (PPT ou polycopié).

Nous nous restreindrons dans ce travail à approcher les comportements de réception des étudiants en CM, à traiter de la manière dont est perçue la complémentarité discursive existant entre le discours oral de l'enseignant en $\mathrm{CM}$ et l'écrit public qu'il projette à son auditoire, et à évaluer modestement l'aide apportée par les TICE, notamment par le biais du bureau virtuel (BV).

\section{Cadre méthodologique}

Cette recherche s'inscrit dans la ligne des travaux menés depuis plusieurs années au sein d'ADIS-langues laboratoire ICAR, Université Lumière Lyon 2 sur l'analyse des CM, qui ont mis en évidence un certain nombre de phénomènes discursifs et contextuels susceptibles d'avoir un impact sur leur réception par les étudiants (Bouchard \& Parpette, 2008) : 
- les différents rôles joués par l'enseignant et la complexité discursive qui en découle,

- le dialogisme et la polyphonie,

- les reformulations et les jeux de posture dans le discours enseignant,

- les différents types de coformulation « discours oral / documents projetés ».

Dans une perspective didactique, les travaux engagés portant sur l'analyse de discours ont de ce fait amorcé la première étape d'une recherche sur l'acquisition des compétences universitaires par les étudiants allophones, et plus largement par l'ensemble des étudiants récepteurs des CM. Le présent travail entend approcher plus spécifiquement les représentations des étudiants sur leurs pratiques en la matière afin de faire avancer la réflexion sur lesdites compétences à acquérir.

Nous reprendrons donc à dessein le terme de «coformulation discursive » (ibid., 2008) précédemment évoqué dans le but de rendre compte de cette présence de deux discours qui coexistent au sein d'un CM avec PPT, en évoquant le type de difficultés que sa gestion entraine auprès du public étudiant. Plus précisément, notre attention se portera sur la manière dont sont perçues ces macro et micro reformulations qui émaillent les performances orales des maîtres de conférences par les auditeurs. Dans la mesure où en effet, «l'oralisation se doit de conserver les caractéristiques globales du discours écrit-visuel (...) sa superstructure et sa macro-structure » (ibid.), l'enseignant en charge d'assurer un cours va utiliser son PPT avec une liberté de parole par rapport à ce qui est écrit plus ou moins importante et appréciée en matière de réception.

\section{Analyse des comportements de réception des CM (avec PPT)}

Le PPT, facteur d'évolution ou de déséquilibre ? Il parait sans doute un peu réducteur de parler «du» PPT considérant la pluralité des idiosyncrasies qui peuvent s'exprimer au travers des pratiques enseignantes. Nous ne les passerons cependant pas en revue, nous centrant davantage sur la manière dont les étudiants s'adaptent à ce qui leur est proposé, en relation avec leurs propres représentations du/des CM en question. A la question ouverte «0221- Quelle est votre impression générale sur cette forme de cours ? », la moitié des étudiants répond positivement mettant en avant une image de modernité, de praticité, d'aide afin d'orienter et de structurer le cours, d'attractivité, d'interactivité, etc. Les réflexions les plus mitigées (30\%) ont trait quant à elles aux difficultés concernant l'écoute simultanée du discours de l'enseignant et la saisie des informations écrites relayées par le PPT. Il est fait fréquemment allusion aussi à des questions de méthodologie qui évoquent des supports surchargés ou non-indispensables en fonction des disciplines abordées (cf. infra). En résumé, le PPT ne séduit pas de facto, s'il semble souvent apprécié, son existence doit être pleinement justifiée par l'utilisation que l'enseignant-concepteur en propose, sinon les comportements « déviants » sont vite repérés : «C'est inutile: le prof n'apporte rien, il lit son cours », « un peu plus vivant, mais un peu «catalogue », « intéressant car montre les points du cours en remplaçant les anciens transparents; mais en même temps terriblement sommaire ou surchargé », " en général, les profs ne savent pas s'en servir », etc.

Mis en situation d'écoute et d'observation synchrones, les étudiants se voient d'emblée confrontés à une activité complexe, cognitive d'abord puisqu'il s'agit avant tout de comprendre une information théorique nouvelle; stratégique ensuite puisqu'il doit sélectionner dans l'ensemble des données fournies par l'enseignant, de façon quasi simultanée, celles dont il conservera une trace matérielle sous forme de notes ; technique enfin pour assurer une prise de notes performante grâce à des procédés de mise en page et d'abréviations efficaces (ibid.).

Ces trois plans (cognition, stratégie et technique) nous offrent ainsi une grille de lecture des représentations des étudiants vis-à-vis de notre objet d'analyse initial.

Sur ce premier aspect lié à l'accès d'un contenu, nous mentionnerons les réactions suscitées par la question suivante «0222- Est-ce que, en général, cela [les présentations PPT] vous aide à mieux comprendre le contenu du cours ? ", qui révèle que 50\% des personnes interrogées se montrent plutôt 
enthousiastes sur le sujet. Ce parti pris est dû essentiellement aux possibilités du PPT en matière d'illustration «en histoire de l'art j'ai pas le choix, pour visualiser les œuvres » ou de synthétisation des informations « on voit mieux où veut en venir le prof». Il est également «plus aisé de noter des citations ou des références lorsqu'on les recopie » selon un étudiant de lettres modernes. $27 \%$ des personnes interrogées se montrent néanmoins franchement réservées quant à l'intérêt du PPT pour accéder plus facilement à ce que veut transmettre l'enseignant, certains déclarant même qu'un PPT surchargé peut être source de démotivation... Pour le reste, la manière dont le PPT est fait et utilisé reste primordiale, celui-ci ne garantissant pas un accès à des informations clairement assimilables.

Ainsi, on peut se poser la question d'un recours systématique au PPT en étayage au CM dans la gestion d'informations nouvelles. Les réactions recueillies permettent en tout cas de remettre en perspective cette pratique en montrant que les possibilités à la fois plurisémiotiques et dynamiques (ibid.) offertes par le PPT restent assujetties à des contextes d'utilisation, des disciplines et des besoins précis.

De manière stratégique ensuite, le recours à l'écrit proposé par l'enseignant grâce au PPT peut permettre d'opérer une discrimination secourable pour l'étudiant perdu dans un flux discursif plus ou moins ininterrompu. Afin de mesurer son efficacité en la matière, nous nous référons ici aux réactions suscitées par cette question: «0228- Que prenez-vous en notes : ce qui est écrit sur le PPT ou ce que dit l'enseignant ? ». Une bonne partie des étudiants $(42,5 \%)$ a évoqué la nette complémentarité entre les deux sources «l'un illustre l'autre », notant que le PPT permettait de présenter des illustrations et de fournir à tous une structure, un «balisage » pour reprendre un terme utilisé de ce qui se dit oralement. Un tiers des interrogés $(34,5 \%)$ dit pourtant préférer suivre en priorité le PPT qui leur est proposé, que ce soit d'ailleurs par défaut : «pas le temps de prendre en note les commentaires de l'enseignant », « [je] recopie le ppt puisque c'est le prof [qui l'a fait]», «[je note] ce qui est écrit sur le ppt donc [je] rate ce qui est dit ») ou de manière plus motivée ( « [avec le] ppt on retient l'essentiel», «je prends pratiquement tout le ppt en note ». Les stratégies déployées montrent de fait que le support en question s'impose en quelque sorte à certains qui le gèrent tant bien que mal, alors que quelques-uns s'attachent à reproduire exactement son contenu en y voyant un condensé des connaissances à retenir. Dans les deux cas, on peut imaginer qu'un polycopié pourrait éventuellement faciliter le travail des premiers, tout en allégeant celui des seconds qui semblent reléguer le discours de l'enseignant à un fond sonore.

Si l'on observe ensuite les motivations des étudiants qui disent se focaliser sur le discours du " prof ", certains regrettent leur propre «tendance à recopier le PPT alors que les commentaires du prof sont aussi nécessaires » et d'autres tranchent ainsi «ce que dit l'enseignant [...] c'est le plus important, le PPT n'étant qu'un résumé du cours ».

Sur un plan technique enfin, il est essentiel pour l'auditeur d'un CM de conserver une trace le plus souvent écrite de ce qui a été dit par l'enseignant. Est-ce que le PPT apporte une aide notable dans ce sens? Pour en mesurer les effets, nous avons pris en compte les réponses apportées aux questions suivantes : «0226- Pensez-vous que vous prenez des notes plus « efficaces» lors d'un cours avec PPT (en comparaison avec d'autres types de cours) ? » et «0227- Pourquoi ? Pouvez-vous commenter votre réponse précédente ?». Si les appréciations ont été très variables, la moitié des personnes interrogées ne remarque pas de différence quant à la prétendue efficacité de la présence d'un PPT en matière de prise de notes : «non, car on se focalise uniquement sur le PPT», «le prof met l'essentiel mais le temps de tout copier ce qui est au tableau et les commentaires du prof $\rightarrow$ les informations sont moins liées », «cela dépend car on se concentre sur le PPT donc on manque une partie », "ce ne sont pas mes phrases, trop synthétiques », «non : piège = recopier juste les grands titres sans prendre le reste du cours », etc. S'ajoutent les remarques plus mitigées de ceux (8\%) qui pensent "possible» l'efficacité du PPT en matière de prise de notes, et enfin celles plus positives des étudiants $(42 \%)$ pour qui le discours de l'enseignant se voit clarifié, structuré, organisé par la planification écrite qu'il en propose par le biais de son PPT : « on se perd dans le CM, permet de rebaliser le cours », " moins d'informations donc on retient l'essentiel », «possibilité de prendre les schémas », etc.

Enfin, selon les disciplines, des usages différents des diapositives projetées au public peuvent bien entendu apparaitre accompagnés par des modalités discursives variables d'un enseignant à l'autre. Sans 
généraliser les représentations, nous nous proposons de rendre compte dans le cadre restreint que propose notre enquête de la manière dont les étudiants apprécient les PPT en CM en fonction de leurs spécialités. En effet, les réponses recueillies à la question «0233- Est-ce que, selon vous, parmi les cours auxquels vous assistez, il y a des «matières » qui se prêtent plus à l'utilisation de TICE (en particulier PPT) que d'autres ? » laissent apparaitre des éléments de réflexion dont on peut se saisir.

On observe ainsi que c'est assez naturellement le rôle de support servant la présentation à un groupe d'un document iconique de type carte, peinture, schéma, graphique qui justifie de prime abord chez les étudiants le recours à un PPT. Dans cette logique, il apparait incontournable qu'en biologie «toutes les matières peuvent s'y prêter », qu'en géographie physique «il est impossible d'imaginer ce dont parle d'enseignant sans » de même qu'en histoire de l'art. Il ressort également que certains étudiants opèrent des distinctions au sein des «matières » qui composent leur cursus, les cataloguant en quelque sorte : «Pas nécessaire en littérature comparée, au contraire en histoire littéraire cela facilite la récupération de certaines informations ${ }^{3}$ », « en économie pour les graphiques, pas utile en droit ou en comptabilité », " en psychologie cognitive c'est justifié mais pas en psychologie clinique ${ }^{4} »$, (en langue) «le PPT n'est pas justifié en thème et version », etc. On voit que les classifications ainsi opérées laissent transparaitre des points de vue concernant l'utilisation des PPT qui tendent à justifier son recours majoritairement lorsque qu'il n'y a pas que du texte, mais des éléments plus visuels en jeu (tableau, schéma, graphiques, etc.).

Dans les disciplines où les objets iconiques sont peu présents telles que les lettres modernes, le droit ou l'anthropologie par exemple, la moitié des interrogés ( 2 sur 4 pour les lettres), ( 1 sur 2 pour le droit) et (2 sur 4 pour l'anthropologie) ne voient même pas l'intérêt d'avoir à suivre un PPT durant leurs CM, leur préférant explicitement le polycopié jugé plus efficace pour la rétention des savoirs enseignés. Autrement dit, il apparait que le PPT, document figé annexé au discours de l'enseignant, qu'il le complète ou s'y substitue selon les méthodes, ne remporte pas une adhésion unanime. Les comportements de réception des étudiants se caractérisent selon nous par une hétérogénéité qui pousse à réinterroger le recours au PPT comme étayage indispensable au discours universitaire.

\section{Une complémentarité discursive problématique}

La singularité du genre discursif d'un $\mathrm{CM}$ avec support écrit tient donc à la bifocalisation à laquelle est invité tout auditeur qui entend suivre le cours avec attention. Celui-ci doit garder le cap de la prise efficace de notes en tentant de transformer en un objet scriptural fiable et économique la performance verbale à laquelle se livre devant lui l'enseignant (Bouchard, Parpette \& Pochard, 2005). Cette opération, nous l'avons observé, n'est pas sans poser de difficultés d'ordre méthodologique et stratégique (quel canal de l'information privilégier ?) mais aussi de nature purement cognitive (qu'est-ce qui retient le plus mon attention?).

Il parait intéressant de mesurer que ces difficultés semblent prendre leur source dans la manière dont les enseignants reformulent oralement le discours figé du PPT. Cette reformulation régie selon une " double contrainte » (Bouchard et Parpette, 2008), impose à la fois similitude et différenciation par rapport à l'énoncé initial. Ainsi, c'est sous forme de « reprises », et "d'ajouts » que l'enseignant commente les items de son PPT, se permettant donc des libertés qui vont être variablement reçues par ses auditeurs. Nous l'avons observé plus haut, si certains préfèrent que l'enseignant utilise l'écrit qu'il projette pour accompagner et structurer fidèlement ses propos, d'autres portent une attention plus appuyée à son discours et à ses gloses y décelant un vecteur de connaissances plus riche.

En résumé, on pourrait dire que suivre un cours qui donne à voir et à entendre de concert ne va pas de soi, et que chaque « récepteur » monopolise selon une logique qui lui est propre des aptitudes lui permettant de s'en sortir. On mentionnera comme étant du domaine de l'anecdote le témoignage de cette étudiante qui dit travailler « à deux » pour la prise de notes en CM, chacun ayant le rôle distinct soit de consigner le contenu du PPT, soit les paroles de l'enseignant... soulignant encore une fois la surcharge cognitive que peut représenter une exposition simultanée à deux sources d'informations. 
De manière complémentaire, on pourrait imaginer que les pratiques de réception des $\mathrm{CM}$ des étudiants (focalisation de l'attention, gestion de la prise de notes, etc.) gagneraient à être davantage aiguillées par les intentions des enseignants si celles-ci s'exprimaient peut-être de manière plus explicite. Nous en voulons pour preuve la multiplicité des remarques qui montrent des stratégies diamétralement opposées quant à la manière d'accueillir ce que l'enseignant propose. Entre ceux qui avouent qu'ils ne savent «pas quoi écrire », ceux qui notent «ce que dit l'enseignant surtout : c'est le plus important, le PPT n'étant qu'un résumé du cours », et enfin les derniers qui prennent « ce qui est écrit sur le PPT : pas le temps de prendre en note les commentaires de l'enseignant", la diversité semble régner quant à la manière de décoder les messages et les intentions de celui qui orchestre un CM. Si l'on peut volontiers reconnaitre que les compétences universitaires sont variables d'un étudiant à l'autre, il serait toutefois souhaitable que les enseignants soient davantage conscients de la portée de leur comportement à l'oral qui peut produire des distorsions dans la communication, des combinaisons oralographiques parfois complexes à aborder.

\section{Le recours aux TICE}

Les étudiants ont été interrogés quant à l'intérêt qu'ils pouvaient entrevoir dans la mise à disposition via des plateformes de cours de type BV soit avant, soit après le cours du PPT élaboré par l'enseignant. Les avis sont partagés puisque $50 \%$ des étudiants ayant répondu à la question (7 sur 14 au total) : 0322- « $\mathrm{Si}$ le PPT est mis à votre disposition avant le cours, en quoi cela change-t-il votre comportement en cours? », pensent effectivement pouvoir consacrer du temps à prendre connaissance du PPT avant le cours. Les arguments évoqués sont les suivants : découverte plus facile du cours ensuite, meilleure concentration favorisée durant celui-ci, le PPT est un bon indicateur pour évaluer l'intérêt même du cours à suivre, et enfin la prise de notes peut s'en trouver plus aisée. La seconde moitié des étudiants qui se montre la plus mitigée sur la question ne semble pas convaincue par l'intérêt de lire la présentation de l'enseignant avant le cours, arguant que cela ferait même doublon avec le cours lui-même, et serait un facteur de démotivation pour y assister.

Cependant lorsque l'on évoque la mise à disposition des étudiants du support projeté durant le $\mathrm{CM}$ après celui-ci (question 0323 en annexe), 61,5\% des personnes interrogées reconnaissent que le PPT peut assister efficacement un travail de révision (permet de compléter, de réordonner les notes) surtout quand des documents iconiques sont intégrés à la présentation (schémas, tableaux, etc.); avoir ainsi la possibilité de retrouver le fil écrit du CM autoriserait une sorte de décharge cognitive durant celui-ci (meilleure concentration sur le discours enseignant). Enfin, les réserves sur la méthode évoquée relativisent quant à elles ce «retravail » effectué par les étudiants a posteriori que permettrait un PPT mis en libre accès.

En définitive, il est intéressant de voir que quel que soit le genre de stratégie d'enseignement déployée (PPT donné avant, projeté en cours ou mis à disposition après), les étudiants ne savent pas toujours précisément comment y répondre avec méthode. Dans un précédent travail de recherche sur la mise en place d'un environnement numérique de type réseau social destiné à l'enseignement/apprentissage d'une langue en contexte universitaire (Dufour \& Grassin, 2012), nous attirions l'attention à l'instar de F. Mangenot (2010) sur le fait que dans les dispositifs en ligne, il y a souvent sous-estimation du rôle de la médiation pédagogique et surestimation de l'autonomie des apprenants. Il s'agit de l'écueil d'une approche centrée sur l'outil plutôt que sur ses usages pédagogiques. Nous pensons qu'il en va de même dans les cas de figure qui nous occupent ici, qu'un outil de médiation de la connaissance comme le PPT n'entraine pas pour autant des usages prescrits et lisibles, et que les étudiants s'en remettent à des comportements quelque peu intuitifs et fluctuants. A ce titre, une piste de réflexion afin d'optimiser une meilleure appropriation par les étudiants des PPT pourrait par exemple consister en l'accomplissement de tâches préparatoires aux cours - questionnaires à remplir, schémas à compléter, etc. - rendant ainsi nécessaire le visionnage du PPT sur le BV pour préparer telle activité (même si ce sont souvent les travaux dirigés en groupes restreints qui prennent en charge la partie plus pratique des enseignements). On ajoutera aussi l'intérêt didactique pour des étudiants allophones que peut constituer la prise de connaissance d'éléments contenus dans un CM avant d'y assister (Bouchard, 2009). 


\section{Conclusion}

En guise de conclusion on reconnaitra que le recours à une version écrite d'un discours oral via une projection publique est désormais un élément connu voire attendu du CM. Si l'on se place du côté des étudiants, cette systématicité reste contestable même si elle rassure, car elle engendre des pratiques servant plus ou moins la compréhension globale du cours tout en ne garantissant pas une prise de notes exhaustive. Il serait donc appréciable que les remarques qui peuvent être recueillies auprès de ceux qui côtoient les bancs des amphithéâtres reviennent aux oreilles des enseignants, que cet écho (méthodologie du PPT à revoir, support non justifié selon certaines disciplines, etc.) serve à parfaire la circulation les échanges au sein du CM.

Pour finir avec une remarque d'un étudiant qui en répondant à la question suivante : Que prenez-vous en notes : ce qui est écrit sur le PPT ou ce que dit l'enseignant? dit « chercher à trouver un équilibre entre les deux », nous pensons que cet objectif tout à fait louable reste néanmoins périlleux, car soumis à trop d'aléas sans même parler de ceux qui attendent des étudiants alloglottes. Il semble donc qu'un champ de recherche s'ouvre sur cette problématique des stratégies de réception des étudiants en $\mathrm{CM}^{5}$, qui devront de manière prospective être croisées avec les représentations et méthodes des enseignants-mêmes qui font et défont cette « culture » universitaire, afin que production et réception en CM résultent progressivement d'une concordance moins instable.

\section{Références bibliographiques}

Bouchard, R. (2009) (sous presse) : «Les appuis iconiques (type powerpoint) pendant les Cours Magistraux : quelle aide à la compréhension pour les étudiants étrangers? », Cahiers $d u C L A$, Besançon.

Bouchard, R. \& Parpette, C. (2008) : «Reformulation et coformulation dans la communication scientifique avec support écrit ", in Schuwer M., Le Bot M.-C., Richard E., (éds.). Pragmatique de la reformulation : types de discours- interactions didactiques, Rennes : Presses universitaires de Rennes, 55-74.

Bouchard, R., Parpette, C. \& Pochard, J-C. (2005) : Le cours magistral et son double, le polycopié : relations et problématique de réception en L2, Cahiers du Français Contemporain 10, Lyon : ENS-Editions, 191-208.

Dufour, S. \& Grassin, J.-F. (2012) : Web social et communauté d'apprentissage en contexte homoglotte. Comment le web 2.0 peut-il modifier les dispositifs et processus d'enseignement / apprentissage dans un centre universitaire en France ?, Actes du 5ème colloque International de l'ADCUEFE, Dijon, 27-28 mai 2011 (article en cours de publication par l'Université de Bourgogne).

Mangenot, F. (2010) : Formation en ligne. Point didactique. Le Français dans le monde n³67 (jan.-fév. 2010), p. 2325.

\section{Annexe}

\section{Questionnaire des entretiens}

\section{1.- Renseignements d'ordre général.}

- Parcours d'études choisi ou intitulé du Master selon le niveau

- Niveau (1, 2, $3^{\text {ème }}$ année, Master 1, Master 2)

- Types de « cours » auxquels l’étudiant est généralement exposé.

(Laisser parler l'étudiant en notant les termes qu'il emploie - cours, TD, cours magistral, etc.). 
02.- Renseignements sur les formes de cours auxquelles le répondant est exposé. (Reprendre la question précédente en faisant préciser la forme de cours).

021.

- TD sans PPT

- TD avec PPT

- TD avec Rétroprojecteur (RP)

- CM vocal (cours magistral sans auxiliaires pédagogiques)

- CM vocal + tableau (quand le tableau est utilisé pour autre chose que l'orthographe d'un mot nouveau)

$-\mathrm{CM}$ vocal + RP

- CM vocal + PPT seulement de l'écrit, plan, résumé, notes, etc.

- CM vocal + PPT images, vidéo, schémas, tableaux, etc. + écrit

022. Réactions aux présentations PPT

Avez-vous déjà eu des cours en PPT ? Oui/non (si non passer à la question 023)

0221- Quelle est votre impression générale sur cette forme de cours?

0222- Est-ce que, en général, cela vous aide à mieux comprendre le contenu du cours ?

0223- Est-ce que vous trouvez que cet apport technologique vous paraît plus attrayant qu'un cours « classique »?

0224- Est-ce que vous avez l'impression de mieux mémoriser ce genre de cours (en comparaison avec d'autres types de cours)?

0225- Est-il plus facile de prendre en notes les cours avec PPT ?

0226- Pensez-vous que vous prenez des notes plus «efficaces» lors d'un cours avec PPT (en comparaison avec d'autres types de cours)?

0227- Pourquoi ? Pouvez-vous commenter votre réponse précédente?

0228- Que prenez-vous en notes : ce qui est écrit sur le PPT ou ce que dit l'enseignant ? Commentez.

0229- Pensez-vous qu'il vous est plus facile d'interrompre l'enseignant lors d'une projection PPT que lors d'un cours normal ? Pouvez-vous expliquez votre réponse? 
023-. Réactions générales à l'emploi des TICE

0231- Parmi tous les modes de présentation des cours (présentés en 0221), lequel à votre préférence ? Pourquoi?

0232- D’une manière générale, est-ce que vous avez l'impression que l'emploi des TICE (liées à l'informatique ou plus traditionnelles, comme le rétroprojecteur ou la vidéo), vous motive/aide plus que des formes de cours strictement orales ? Pourquoi ?

0233- Est-ce que, selon vous, parmi les cours auxquels vous assistez, il y a des « matières » qui se prêtent plus à l'utilisation de TICE (en particulier PPT) que d'autres ? Pouvez-vous commenter un peu votre réponse?

\section{3.- Stratégies étudiantes pendant les cours}

031- Que faites-vous pendant un cours « normal»?

0312- Quand vous prenez des notes, comment les utilisez-vous par la suite?

0313- Vous arrive-t-il de vous rendre compte que vos notes sont difficilement utilisables ? (à expliciter)

0314- Comment remédiez-vous à ce problème?

0315- Si vous avez déjà utilisé pour une raison ou une autre des notes prises par vos camarades, quelles difficultés d'utilisation avez-vous rencontrées ?

032- Si vous avez déjà été confronté(e) à une présentation PPT.

0321- est-ce que cela a changé votre façon de prendre des notes ou plus généralement votre comportement en cours.

0322- Si le PPT est mis à votre disposition avant le cours, en quoi cela change-t-il votre comportement en cours?

0323- Si le PPT est mis à votre disposition après le cours, en quoi cela change-t-il votre comportement ? Explicitez et indiquez comment vous travaillez à partir de ce document.

0324- Si vous avez déjà eu l'expérience d'un polycopié accompagnant le cours, que préférez-vous le polycopié ou le PPT ? Explicitez.

Merci pour votre collaboration. 


\footnotetext{
${ }^{1}$ Technologies de l'Information et de la Communication pour l'Enseignement.

${ }^{2}$ Cf. "Les environnements numériques dans les cours universitaires : objectifs des enseignants, usages des étudiants", intervention en collaboration avec C. Parpette, Colloque International "Langages - Cultures - Sociétés : Interrogations didactiques", Université Sorbonne Nouvelle - Paris 3 (DILTEC), 20-22 juin 2012.

${ }^{3}$ Nous n'avons ici pas les données précises sur la nature des informations en question (frises chronologiques, listes de dates, tableaux, etc. ?).

${ }^{4}$ Même remarque que pour la note précédente.

${ }^{5}$ Travaux du groupe de recherche « français sur objectif universitaire » conduit par C. Parpette, laboratoire ICAR, équipe Adis-Langue, Université Lumière Lyon 2.
} 